\title{
HIGH TIME RESOLUTION FHOTOMETRY OF RED DWARF FLARE STARS II. THE SHORTEST FLARE RISE TIME
}

\author{
G.M. Eestin, V.L.FIalihotnichenl:o, \\ L.A.Fustil nit, V.F.Shvartsman
}

Special Astrophysical Observatory Nizhnij Arlthyz, \$57147 USSFi

\section{Fi. E. Gershberg}

Crimean Astrophysical Dbservatory Crimea, Nauchny S\$441S USSF

ABSTRACT. We have studied the initial phases of 65 optical flares on UV cet type stars with a time resolution of 3x10-7 5 . Ninety per cent of these flares have rapid brightenings shorter than 105 and several are shorter than 15 . We detected on UV Cat the shortest flare observed to dite with a total duration of $1.7 \mathrm{~s}$ and a rise time of 0.35 . The shortest flare rise times on the observed stars fit the predictions of the gas-dynamic flare model by ratsova et al. (1981) and ratsova and Livshits 1984).

We selected ts flares with well observed initial phases froin a set of $7 \Xi f l a r e s$ on CN Leo, UV Cet, Wolf 424 and $V 577$ Mon. The observations were obtained with $3 \times 10^{-2} 5$ time resolution during the course of our flare star monitoring projran at the $b^{-m}$ telescope of the Special Astrophysical Dbservatory using the MANIA system (Neizvestnyj and Fimonov, 1978; Fimanov, 1979). During the rising phase of a stellar flare the continuun radiation dominates in the optical wavelength range (Bopp and Moffett, 197ङ) and just at this phase one may expect to find some effects of non-thermal processes.

For each of the ss selected flares we measured the duration of the "leading front", i.e., the duration of the phase when the flare intensity increases with the steepest and nearly constant rate. We excluded the slow brightness increases sometimes observed at flare beginning, which are due to emission line radiation (Bopp and Moffett, 1973).

The distributions of leading front durations are given in figure 1. These plots show that for the overwhelming majority of flares tiiese durations do not exceed $10 \mathrm{~s}$. There may be a maximum near 2- 5 that is more clearly exhibited in the total distribution, which includes all four ahserved stars. Dn each of these 5 tars we detected flares with leading front durations shorter than $1 s$. The light curves of these flares are shown in figure 2 . The UV Cet flare on 18 December 1984 , at $18 \mathrm{~h} 09 \mathrm{~m}$ UT, was the most $r$ apid event ever detected: the flaring $u_{\mu}$ time and total duration were 0.35 and $1.7 \mathrm{~s}$, respectively. 


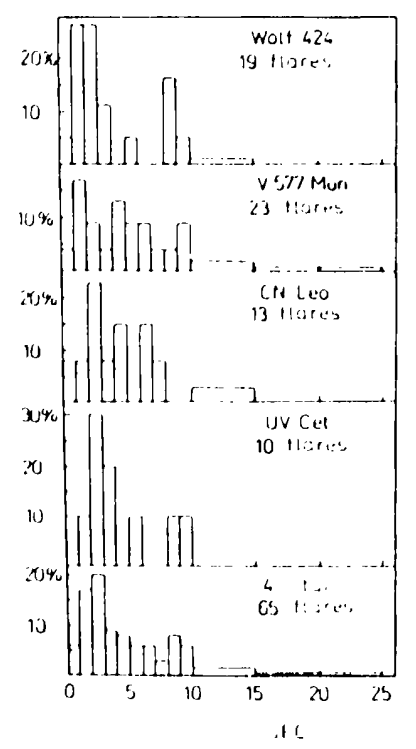

Figure 1. Distributions of leading front durations for stellar flares.

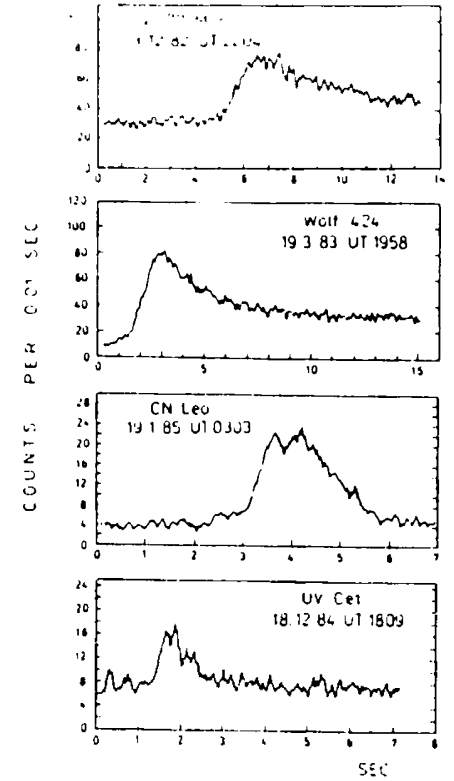

Figure 2. Light curves of stellar flares with fast initial phases.

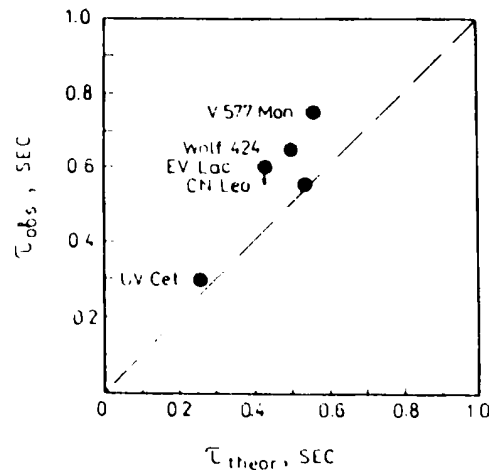

Figure $3 . \quad$ Comparison between the shortest flare leading fronts from observations and theoretical estimates $\left(r_{m+n}\right)$ from the gasdynamical stellar flare model. 
Dne can show that if the optical flare radiation is due to nonthermal -mechanisms, flares may have fine structures on time scales in the range $10^{-0}-10^{-1} 5$, while for thermal mechanisms significant details on flare light curves must exceed $0.1-15$. Therefore, the fact that the rise times for all of the observed events exceed $0.2-0.35$ implies that there is no evidence that the optical flare radiation is excited by a non-thermal mechanism, even at the very beginning of flares.

Within the frameworl of the thermal gas-dynamic model of flares on UV Cet type stars (Katsova et al., 1981; Katsova and Livshits, 1986), the lifetime of the rising phase is ultimately determined by the time interval to shift the front of a shock wave that propagates along a magnetic flux tube, toward the stellar photosphere, i.e., to a distance of about one scale height of the stellar atmosphere. According to this model, $\tau_{m x n}=V_{0} / M g$, where $v_{m}$ is the sound velocity in the low quiet chromosphere, $M$ is the Mach number for the downward shock wave and 9 is the gravity acceleration at the stellar surface. We have used Fiodono's (1987) g data for $f l$ are stars and the estimate of $M$ ? 30 by Katsova and Livshits (1986) to calculate rim for the flare stars in Fig. 2 and for EV Lac, a very rapid flare of which was detected with a temporal resolution 0.315 with the space astrophysical station ASTFON (Gershberg and Fetrov, 1986). In Fig. $\Xi$ the computed $\tau_{m i n}$ are compared with the observed durations of the leading fronts. For EV Lac we can estimate only the upper limit of such duration. Fig. S shows that the observed times are rather close, but never shorter than the theoretical limits. This fact is an argument in favour of the validity of the thermal gas-dynamic model for stellar flares on uU cet type stars.

The complete version of this study is published in the Letters to Astron. J. (USSF) 14, No. $3,1988$.

\section{FEFERENCES}

Eopp B.W. Moffett T.J.: 1973, Astrophys. J. 185, 239.

Gershberg R.E, Petrov F.P.: 1986, in "Flare stars and related objects", L.V.Mirzayan (ed), Fubl. ficad. Sci. Armenian SSFi, Erevan, p. 38.

Katsova M.M., Kosovichev A.G., Livshits M.A.: 1981, Astrofizika 17,285 .

Catsova M.M., Livshits M.A.: 1986, in "Flare stars and related objects", L.V.Mirzayan (ed), Fubl. Acad. Sci. Armenian SGR, Erevan, p. 18?.

Neizvestnyj S.I., Fimonov A.A.: 1978, Comm. Special Astrophys. 0b5. $23,5 t$.

Fimanov A.A.: 1979. Comm. Special Astrophys. 0b5. 25, 31.

Fodond M.: 1987, in "M-type stars", H.R. Johnson and F.R. Querci (eds), CNFiG-NASA Monograph Series on Nonthermal Phenomena in Stellar Atmospheres, NASA SP-492, p. 409. 